\title{
Caracterización Reológica del Suero Costeño de Turbaco, Arjona, El Carmen de Bolívar y uno Comercial (Colombia)
}

\author{
Diofanor Acevedo ${ }^{(1)^{*}}$, Clemente Granados ${ }^{(1)}$ y Ramiro Torres ${ }^{(2)}$ \\ (1) Universidad de Cartagena, Facultad de Ingeniería, Programa de ingeniería de Alimentos, Avenida el \\ Consulado, Calle 30 No. 48-152. Cartagena, Bolívar-Colombia (e-mail:diofanor3000@gmail.com). \\ (2) Universidad de Córdoba, Facultad de Ciencias Agrícolas, Departamento de Ingeniería de Alimentos, \\ Carrera 6 No 76-103, Km. 3, vía Cereté, Córdoba-Colombia. \\ * autor a quien debe ser dirigida la correspondencia
}

Recibido Sep. 16, 2013; Aceptado Oct. 21, 2013; Versión final recibida Nov. 9, 2013

\begin{abstract}
Resumen
El objetivo de esta investigación fue determinar los efectos de las condiciones de proceso sobre las propiedades viscoelásticas del suero costeño elaborado en los municipios de Turbaco, Arjona y El Carmen de Bolívar (Colombia), y compararlas con las de un suero costeño comercial. Se determinó el módulo elástico, la viscosidad compleja (Pa.s) y la tangente de perdida $(\tan \delta)$ en los diferentes sueros costeños, utilizando un reómetro TA AR 1500, ajustando los datos de viscosidad compleja a los modelos de Potencia, Ellis, Cross y Cross-Carreau. Los resultados mostraron que los modelos que mejor describen el comportamiento viscoelástico del suero costeño son los de Ellis y Cross, y que el tiempo de fermentación incide inversamente sobre su consistencia. El suero costeño más viscoso fue el de El Carmen de Bolívar $(370,50)$, seguido del Comercial $(229,86)$, Arjona $(74,05)$ y Turbaco $(68,06)$. Todos los sueros costeños estudiados tienen un comportamiento pseudoplástico.
\end{abstract}

Palabras clave: suero costeño, propiedades viscoelásticas, módulo elástico, viscosidad compleja

\section{Rheological characterization of a Fermented Milk Product known as Suero Costeño from Turbaco, Arjona, El Carmen de Bolívar and a Commercial Product (Colombia)}

\begin{abstract}
The objective of this research was to determine the effects of process conditions on the viscoelastic properties of the a fermented milk product known as suero costeño produced in the municipalities of the Turbaco, Arjona and El Carmen de Bolivar (Colombia), and compare them with those of a commercial suero costeño. The elastic modulus, the complex viscosity (Pa.s) and the loss tangent (tan $\delta)$ were determined for all samples of suero costeño. For this, a TA AR 1500 rheometer was used and data was fitted to the complex viscosity power models, Ellis, Cross and Cross-Carreau. The results showed that the models that best describe the viscoelastic behavior of the suero costeño are the Ellis and Cross, and that the fermentation time inversely affects its consistency. The most viscous suero costeño was El Carmen de Bolivar $(370,50)$, followed by commercial $(229,86)$, Arjona $(74.05)$ and Turbaco $(68,06)$. All samples of suero costeño presented a pseudoplastic behavior.
\end{abstract}

Keywords: suero costeño, viscoelastic properties, elastic modulus, complex viscosity. 


\section{INTRODUCCIÓN}

El Suero Costeño es un producto lácteo fermentado elaborado tradicionalmente en la Costa Caribe Colombiana, especialmente en la mayoría de los municipios de los departamentos de Bolívar, Sucre, Córdoba y Cesar. También se elabora en algunos municipios de los departamentos de Santander y Norte de Santander debido a la influencia que tienen de la Costa Norte (Granados et al., 2009; Acevedo et al., 2010; Granados et al., 2012). El producto final es similar a la crema agria, un poco ácida y generalmente es usado como aderezo, acompañando casi todas las comidas (Acevedo et al., 2010; Granados et al., 2012). Aunque este se produce industrialmente, presenta un bajo consumo debido a la diferencia detectada por los consumidores en el sabor y a la presencia de sinéresis. Estos problemas de calidad se han tratado de resolver mediante el aumento del tiempo de fermentación y la adición de espesantes, lo cual aumenta los costos y hace más perecedero el producto (Acevedo et al., 2010; Granados et al., 2012).

El Suero Costeño, al igual que muchos alimentos, incluye en su composición una matriz sólida y una fase líquida que aporta un elemento viscoso a la estructura total. Estos alimentos presentan un comportamiento reológico que combina características propias de los sólidos elásticos y de los líquidos, y se denomina viscoelástico (Sahin y Gülüm, 2006)( Acevedo et al 2010). La demanda creciente de los consumidores hacia productos más naturales que contengan menos o ningún aditivo o estabilizantes ha motivado la investigación del uso de componentes de la misma leche o el control de las variables de proceso para mejorar los productos lácteos y evitar problemas como la sinéresis, textura excesivamente blanda y poco tiempo de conservación (Ramírez, 2008).Por otro lado el conocimiento de las propiedades viscoelásticas, además de ser de gran valor para el control de calidad (Schramm, 2000), también es muy útil en el diseño y predicción de la estabilidad de muestras almacenadas (Tabilo-Munizaga y Barbosa-Cánovas, 2005).

Uno de los métodos frecuentemente usado para caracterizar la viscoelasticidad de los fluidos alimenticios son los ensayos de reología dinámica, como los geles son materiales viscoelásticos, las pruebas para evaluar las características de estos sistemas utilizan las propiedades reológicas dinámicas, las cuales suministran información de la estabilidad, fuerza del gel y calidad del producto, esté tipo de información no puede ser obtenida por los equipos de rotación estándar (viscosímetros)

En los ensayos de reología dinámica se aplica de forma oscilatoria una pequeña deformación o velocidad de deformación sobre un fluido y se mide la amplitud de la respuesta del esfuerzo cortante y del ángulo de fase entre el esfuerzo cortante y la deformación. Este ensayo corresponderá al estado de viscoelasticidad lineal si el esfuerzo es linealmente proporcional a la deformación aplicada y si la respuesta del esfuerzo es en forma de una onda sinusoidal (Barnes, 2000). En el caso de un sólido elástico esta onda está en fase con la velocidad de deformación aplicada. Para un líquido viscoso ideal existe un desfase de $90^{\circ}$ entre ellos. En fluidos viscoelásticos el ángulo de desfase está comprendido entre 0 y $90^{\circ}$. Este tipo de ensayo permite determinar la proporción entre el componente elástico y viscoso de un material y cuantificar en qué medida se comporta como sólido o como líquido, a través de funciones viscoelásticas teles como el modulo complejo $\mathrm{G}^{*}$ definido como (Chhabra y Richardson, 2008):

$G^{*}=\sqrt{G^{\prime 2}+G^{\prime \prime 2}}$

Donde G'es el módulo de almacenamiento y representa la componente elástica del producto y G" es el módulo de pérdidas y representa su carácter viscoso. Estos módulos se determinan de la siguiente manera:

$$
\begin{aligned}
& G^{\prime}=\left(\frac{\tau_{0}}{\gamma_{0}}\right) \cos \delta \\
& G^{\prime \prime}=\left(\frac{\tau_{0}}{\gamma_{0}}\right) \operatorname{sen} \delta
\end{aligned}
$$

Donde $\tau_{\circ}$ y $\gamma_{0}$ son, respectivamente, las amplitudes de las ondas del esfuerzo y de la deformación y $\delta$ es el ángulo de desfasaje. Si el fluido es puramente elástico, $\delta=0^{\circ}$ y $G^{*}=G^{\prime}, G^{\prime \prime}=0$. En un fluido viscoso ideal $\delta=90^{\circ}$ y $G^{\prime \prime}=G^{*}, G^{\prime}=0$ (Chhabra y Richardson, 2008). Otras funciones viscoelásticas son el módulo de viscosidad compleja|n*|y la tangente del ángulo de fase $\tan \delta$, usada para describir el comportamiento viscoelástico (Freeman, 1992). Estas funciones viscoelásticas han sido usadas para caracterizar algunos tipos de alimentos tales como: Gel de arroz (Jena y Bhattacharya, 2003), carnes de pescado (Campo y Tovar, 2008), mantequilla (Shukla y Rizvi, 1995), derivados de yema de huevos (Miranda et al., 2000), yogurt y gel de queso (Lucey et al., 1998). 
Este trabajo tuvo como objetivo determinar los efectos de las condiciones de procesos sobre las propiedades viscoelásticas del Suero Costeño elaborado en los municipios de Turbaco, Arjona y El Carmen de Bolívar y el Suero Costeño comercial.

\section{METODOLOGÍA}

\section{Procedimiento de elaboración de Suero Costeño}

Se realizaron visitas a los sitios de producción de Suero Costeño en los municipios de Turbaco, Arjona y El Carmen de Bolívar (Colombia) y a una planta de procesamiento de leche comercial. Con base en entrevistas, inspección y seguimiento a los procesos de elaboración del Suero, se documentaron las formas de elaboración en cada municipio. Se encontraron dos sistemas de producción del suero costeño diferentes: Uno que emplea como materia prima de partida leche entera (Sueros de El Carmen de Bolívar y comercial) y otro que emplea como materia prima de partida el lactosuero, también llamado espiche, subproducto de la fabricación de queso (Sueros de Arjona y Turbaco).Se describen a continuación.

\section{Proceso de elaboración artesanal del Suero Costeño de El Carmen de Bolívar}

Se hace la recepción de la leche, se filtra y se deja en reposo, se fermenta con la flora microbiana propia de la leche durante 7 horas. Después se mezcla y se esperan 10 horas, inmediatamente se filtra para separar el espiche y el coagulo o parte semisólida. Al coagulo se le agrega sal, agua y se bate. Finalmente se almacena $\mathrm{a}-5^{\circ} \mathrm{C}$. Cuando se va a envasar se agrega un poco más de espiche de queso y se envasa.

\section{Proceso de la elaboración artesanal del Suero Costeño de Arjona}

La leche se filtró con coladores, se depositó en tanques plásticos y se le adiciona cuajo líquido. Luego de treinta minutos se separa el espiche o lactosuero dulce dando lugar a la masa de queso. Posteriormente se realizó el salado y amasado de la masa. Del amasado se produce espiche y masa de queso. El espiche se envasa y fermenta durante 18 horas, luego se separa la parte liquida de la semisólida o Suero Costeño final. El Suero costeño final se envasa en botellas, se transporta y distribuye en la zona.

\section{Descripción del proceso artesanal de elaboración del Suero Costeño de Turbaco}

La leche se filtra con un lienzo o tela. Se deposita en tanques plásticos y se le adiciona cuajo. Luego de treinta minutos se quiebra la cuajada y filtra, produciendo masa de queso y el lactosuero dulce. Posteriormente se realiza el salado y mezclado, luego se prensa para obtener queso y espiche salado. Este espiche es fermentado durante 18 horas para obtener el Suero Costeño.

\section{Descripción del proceso de elaboración del Suero Costeño de la planta procesadora}

Se realiza la recepción de la leche. Se toman muestras para hacerles análisis fisicoquímicos y microbiológicos. Después se filtra y se almacena en tanques, a temperaturas entre 4 y $8^{\circ} \mathrm{C}$. Luego se homogeniza a una presión de 10,34 MPa. Se pasteriza de $81-82^{\circ} \mathrm{C}$ por un periodo de 15 minutos. Después se adiciona Cloruro de Sodio para aumentar el rendimiento del producto final. Se agrega el cultivo iniciador y se incuba por un tiempo de 12 a 16 horas. Pasado este tiempo se filtra, separando la parte liquida 0 lactosuero de la semisólida o coagulo, esta parte semisólida se le añade conservantes y sal para darle el sabor al producto. Posteriormente se envasa en bolsas plásticas y se almacena a $4{ }^{\circ} \mathrm{C}$ por 24 horas. Se hace control de calidad para posteriormente liberar el producto que se va a comercializar.

\section{Caracterización viscoelástica}

Se tomaron tres muestras de $500 \mathrm{~cm}^{3}$ de Suero Costeño producidos artesanalmente en los municipios de Turbaco, Arjona y El Carmen de Bolívar (Colombia) y muestras comerciales de Suero Costeño. Las medidas viscoelásticas se realizaron en un reómetro TA AR $1500^{\circledR}$ (TA Instruments Ltda.) utilizando platos concéntricos de $40 \mathrm{~mm}$. Todas las muestras se quedaron en reposo durante 15 minutos para permitir la relajación de las mismas antes de realizar el ensayo.

A los diferentes Sueros costeños se le realizó unos ensayos de barrido de deformación, para elegir el valor de la deformación (en el rango de viscoelásticidad lineal), a la cual se realizó el barrido de frecuencia en un rango entre 0,1 a $10 \mathrm{~Hz}$. La temperatura de las muestras se mantuvo a $25 \pm 0,1^{\circ} \mathrm{C}$. Los ensayos fueron automáticamente controlado por el programa TA Universal Analysis Versión $5.2^{\circledR i n s t a l a d o ~ e n ~ u n ~ o r d e n a d o r ~}$ PC conectado al reómetro. El análisis de datos reológico se realizó utilizando el software Rheology Advantage Data Analysis Version5. $7^{\circledR}$. Con los ensayos oscilatorios se determinaron los módulos de almacenamiento $\left(G^{\prime}\right)$ y de pérdidas ( $G$ "); las viscosidades complejas, $\left(\eta^{*}\right)$ y los valores de la tangente del ángulo de fase (tan $\delta)$, en función de frecuencia de la deformación. 
También se ajustaron los datos experimentales a los modelos Potencia (ecuación 4), Ellis (ecuación 5), Cross (ecuación 6) y Cross-Carreau (ecuación 7); verificando en todo caso que se cumple la ecuación de Cox-Merz (ecuación 8), que relaciona viscosidad dinámica $(\eta)$ con viscosidad compleja $\left(\eta^{*}\right)$. La ventaja de esta relación de la Cox-Merz es que es más simple trabajar con frecuencias ( $\omega)$ que con velocidades de cizallamiento $(\dot{\gamma})$, ya que algunos alimentos o soluciones poliméricas no pueden ser medidos a velocidades de cizallapor encima de $50 \mathrm{~s}^{-1} \mathrm{en}$ un viscosímetro rotacional debido a los efectos elásticos (efecto Weissenberg). Así, en lugar de la medición de una curva de flujo en cizalla de estado estacionario, se puede utilizar más fácilmente la viscosidad compleja de las pruebas dinámicas (Schramm, 2000).

$\eta=k(\dot{\gamma})^{n-1}$

$\eta=\frac{1}{\left[\frac{1}{\eta_{0}}+m^{-1}(\dot{\gamma})^{1-n}\right]}$

$\eta=\frac{\eta_{0}}{1+\left(\frac{\eta_{0} \dot{\gamma}}{\tau}\right)^{1-n}}$

$\eta=\frac{\eta_{0}}{\left[1+\left(\frac{\eta_{0} \dot{\gamma}}{\tau}\right)^{a}\right]^{\frac{1-n}{a}}}$

$\eta(\dot{\gamma})=\eta^{*}(\omega) \rightarrow \dot{\gamma} \approx \omega$

\section{Diseño experimental y análisis estadístico}

Se realizó un diseño completamente al azar (DCA) con el factor en 4 niveles y como variable de respuesta los módulos de almacenamiento, módulo de pérdidas y viscosidad compleja, con 3 repeticiones para un total de 12 unidades experimentales. Se realizó un análisis de varianza y un test de Tukey con un nivel de significancia del $5 \%$, a la variable respuesta viscosidad compleja en las frecuencias $0,1,1,0$ y $10 \mathrm{~Hz}$ para determinar la influencia del tipo de Suero en el comportamiento viscoelástico. El análisis estadístico de los datos se realizó con el programa STATISTICA versión de prueba 8.0.En la evaluación de los modelos se tuvo en cuenta el coeficiente de determinación $r^{2}$ y el análisis de los residuales

\section{ANÁLISIS DE RESULTADOS}

\section{Análisis procesos de elaboración de los Sueros Costeños}

Los procesos de elaboración de Suero Costeño de Arjona, Turbaco y El Carmen de Bolívar son diferentes al de suero comercial ya que en la planta procesadora el proceso consta de una etapa de pasterización y de homogenizado, lo cual aumenta la estabilidad de la leche(Lucey et al., 1998).En la elaboración de los Sueros Costeños del Carmen de Bolívar y comercial la leche es fermentada, mientras que los sueros costeños de Arjona y Turbaco se producen por fermentación del lacto Suero o espiche del queso.

\section{Viscoelasticidad}

La Figura 1 presenta los valores del módulo de almacenamiento y del módulo de pérdida a la temperatura de $25^{\circ} \mathrm{C}$ en función de la frecuencia para los sueros costeños estudiados. Se puede observar que existen diferencias cuantitativas en el espectro para las magnitudes de los parámetros viscoelásticos de los diferentes sueros, y estas se mantienen casi constantes a lo largo de todo el rango de frecuencia estudiada. El comportamiento elástico (módulo de almacenamiento, G') predomina sobre el comportamiento viscoso (módulo de pérdidas G"), también se aprecia el incremento en ambos módulos al ir aumentando la frecuencia siguiendo un comportamiento constante, por lo que todos los Sueros costeños se comportan como un semisólido en todo el rango de frecuencia estudiada. Para los sueros de Arjona y Turbaco, se observa menor variación entre los valores G' y G" con la frecuencia, que para los sueros comercial y del Carmen de Bolívar, teniendo estos últimos los mayores valores de los módulos G' y G", presentando una estructura que requiere de mayores esfuerzos cortantes, para su deformación, lo cual se puede explicar , por la formación de un gel por las caseínas de la leche ,el cual atrapa la grasa mientras los sueros Costeños de Arjona y Turbaco se obtienen como un subproducto de la elaboración del queso y su porcentaje de proteínas es bajo formando un gel débil. Estos valores son semejantes a los reportados para yogurt (Lucey et al., 1998), pulpa de membrillo (Ramos y lbarz, 2006), dispersiones de pectinas (Da Silva et al., 1993) y con mayonesa (Ibarz y Ortiz, 1993).(Granados el al 2012) 


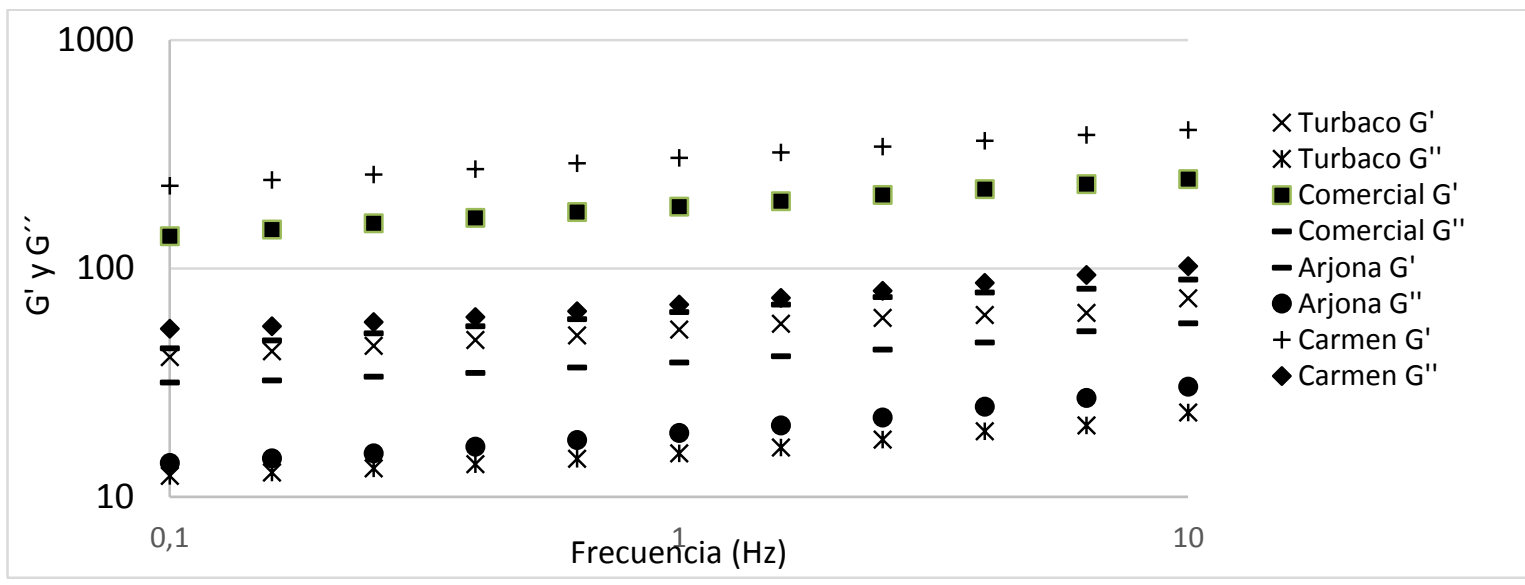

Fig. 1: Módulo de almacenamiento (G') y del módulo de pérdidas (G") en función de la frecuencia para los Sueros Costeños de Turbaco, Arjona, El Carmen de Bolívar y Comercial, a $\mathrm{T}=25^{\circ} \mathrm{C}$.

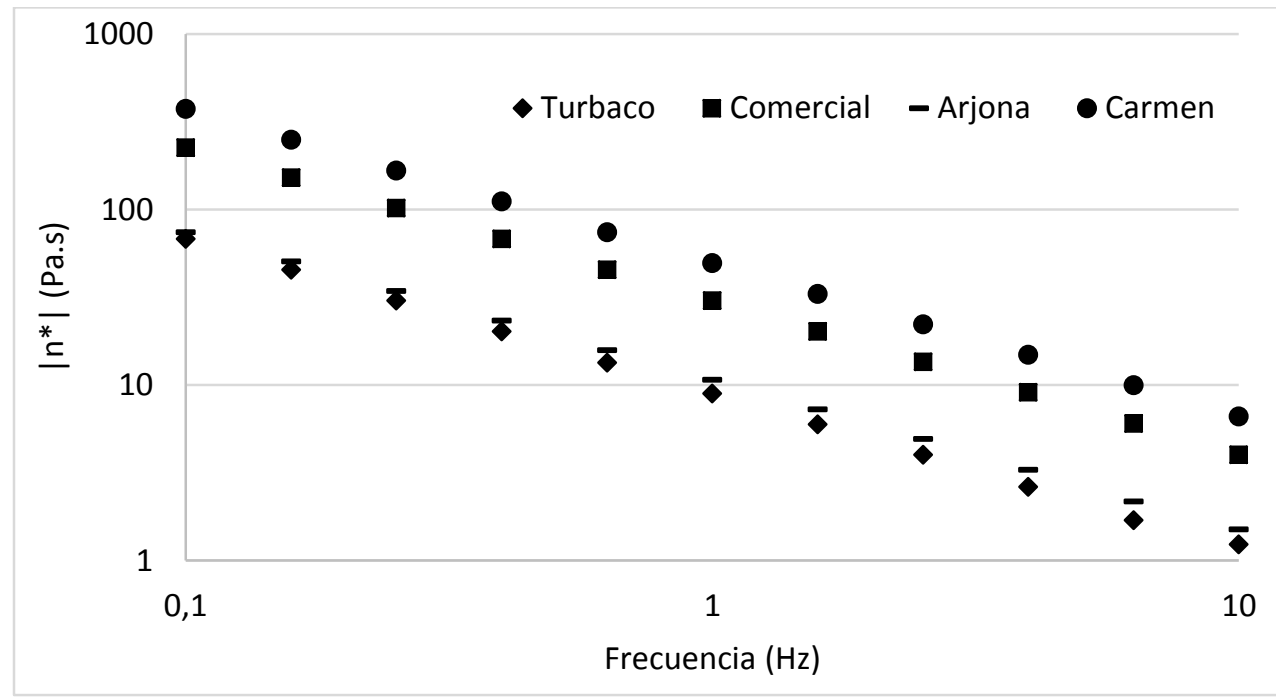

Figura 2. Valores de las viscosidades complejas en función de la frecuencia para los Sueros Costeños de Turbaco, Arjona, El Carmen de Bolívar y Comercial, a $\mathrm{T}=25^{\circ} \mathrm{C}$.

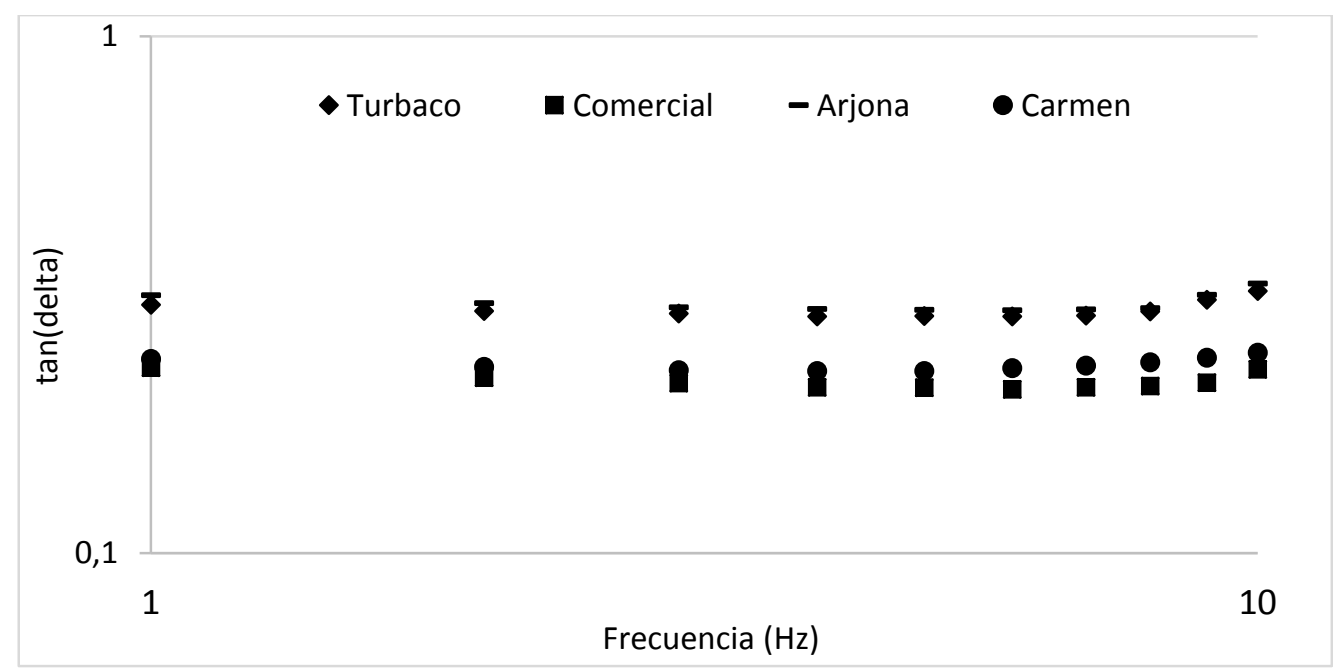

Fig. 3: Valores de la tangente del ángulo de fase $(\delta)$ en función de la frecuencia para los Sueros Costeños de Turbaco, Arjona, El Carmen de Bolívar y Comercial, a $\mathrm{T}=25^{\circ} \mathrm{C}$. 
Como se observa en la Figura 2, el suero de Carmen de Bolívar es el que presenta mayor viscosidad compleja en todo el rango de frecuencia estudiado, seguido del comercial, y Arjona siendo el de Turbaco el que presentó menor viscosidad compleja, lo cual indica la formación geles más fuertes en los sueros del Carmen de Bolívar presentando entonces una mayor comportamiento viscoelastico, incluso por encima del suero comercial debido a sus valores más alto de sus módulos elástico G' y de pérdidas G".

Los valores de la tangente del ángulo de fase, ángulo $\delta,(\operatorname{Tan} \delta)=G$ "'G', para los Sueros costeños estudiados están representados en la Figura 3. La Tanó es una medida adimensional que compara la cantidad de energía perdida durante un ensayo oscilatorio con la cantidad de energía almacenada durante este periodo, e indica si predomina la propiedad elástica o viscosa. Como puede apreciar los Sueros Costeños de Arjona y Turbaco tienen mayor tangente del ángulo de fase en todo el rango de frecuencia que los Sueros costeños de El Carmen de Bolívar y Comercial y este último es el que tiene la tangente del ángulo de fase más baja lo que indica que predomina las propiedades elásticas, probablemente las caseínas forman con la grasa y proteínas del lacto suero estructuras más complejas, como producto de la pasterización, lo cual acentúa el carácter viscoelástico (Lucey et al., 1998),

\section{Modelos de viscosidad}

Debido a que la viscosidad compleja resulto igual a la aparente, en todo el intervalo de velocidad de deformación y frecuencia, la regla de Cox-Merz (Bird y col., 1987), Ec. (8), es aplicable al comportamiento en cizalla estacionaria y no estacionaria como sucede con disoluciones poliméricas isotrópicas comunes. Los modelos de Ellis y Cross fueron los que mejor se ajustaron a los datos experimentales del Suero Costeño comercial con igual errores estándar $(0,4705)$, el modelo de Cross-Carreau aunque tuvo un buen ajuste, presentó un mayor error estándar $(1,273)$, mientras que el modelo de potencia no tuvo un buen ajuste pues el error estándar fue de 54,5, igual comportamiento tuvieron los demás Sueros (Tablas 1 y 2) El índice de comportamiento de flujo describe un comportamiento pseudoplástico, presentando el Suero Comercial el menor valor. Por otra parte los Sueros de Turbaco y Arjona presentaron los índices de consistencia más bajo en comparación con el Comercial y El Carmen de Bolívar que fue el que mayor índice de consistencia presento, concordando el análisis de los mayores valores de los módulos (G' y G").

Tabla 1: Ajuste de los valores de viscosidad compleja de diferentes Sueros Costeños a los modelos de la Ley de la Potencia y Ellis para $\omega=0,1-10 \mathrm{~Hz}$. E.E.: error estándar del ajuste.

\begin{tabular}{|c|c|c|c|c|}
\hline \multirow{2}{*}{ Parámetros } & \multicolumn{4}{|c|}{ Ley Potencia } \\
\cline { 2 - 5 } & Turbaco & Arjona & El Carmen de Bolívar & Comercial \\
\hline $\mathrm{k}(\mathrm{kPa} . \mathrm{s})^{\mathrm{n}}$ & 2,587 & 3,178 & 11,47 & 6,472 \\
\hline $\mathrm{n}$ & 0,031 & 0,032 & 0,024 & 0,022 \\
\hline $\mathrm{E} . \mathrm{E}$ & 66,28 & 81,97 & 49,87 & 54,4 \\
\hline \multirow{2}{*}{ Parámetros } & \multicolumn{4}{|c|}{ Ellis } \\
\cline { 2 - 5 } & Turbaco & Arjona & El Carmen de Bolívar & Comercial \\
\hline no (Pa.s) & 24280 & 3259 & 61610 & 7093 \\
\hline $\mathrm{m}(\mathrm{kPa} . \mathrm{s})^{\mathrm{n}}$ & 45,48 & 51,48 & 251,6 & 155,3 \\
\hline $\mathrm{n}$ & 0,116 & 0,147 & 0,119 & 0,115 \\
\hline $\mathrm{E} . \mathrm{E}$ & 0,5481 & 0,8177 & 0,2811 & 0,4705 \\
\hline
\end{tabular}

Tabla2: Ajuste de los valores de viscosidad compleja de diferentes Sueros Costeños a los modelos de Cross y Cross-Carreau para $\omega=0,1-10 \mathrm{~Hz}$. E.E: error estándar del ajuste.

\begin{tabular}{|c|c|c|c|c|}
\hline \multirow{2}{*}{ Parámetros } & \multicolumn{4}{|c|}{ Cross } \\
\cline { 2 - 5 } & Turbaco & Arjona & El Carmen de Bolívar & Comercial \\
\hline$\eta_{0}(\mathrm{~Pa} . \mathrm{s})$ & 22200 & 3255 & 61320 & 7098 \\
\hline $\mathrm{T}_{0}(\mathrm{~Pa})$ & 20,09 & 25,13 & 119,2 & 94,36 \\
\hline $\mathrm{n}$ & 0,116 & 0,147 & 0,119 & 0,115 \\
\hline E.E & 0,5482 & 0,8177 & 0,2811 & 0,4705 \\
\hline \multirow{2}{*}{ Parámetros } & \multicolumn{3}{|c|}{ Cross-Carreau } \\
\cline { 2 - 5 } & Turbaco & Arjona & El Carmen de Bolívar & Comercial \\
\hline $\mathrm{n}_{0}(\mathrm{~Pa} . \mathrm{s})$ & 385,1 & 134,8 & 707,3 & 323,8 \\
\hline $\mathrm{T}_{\mathrm{o}}(\mathrm{Pa})$ & 34,09 & 42,12 & 216,7 & 135,3 \\
\hline $\mathrm{a}$ & 107,9 & 38,24 & 56,12 & 52,6 \\
\hline $\mathrm{n}$ & 0,118 & 0,157 & 0,122 & 0,129 \\
\hline E.E & 0,5575 & 1,182 & 0,3587 & 1,273 \\
\hline
\end{tabular}




\section{Incidencia del tipo de Suero costeño en el comportamiento viscoelástico}

El análisis de varianza de los diferentes tipos desuero, sobre la variable respuesta viscosidad compleja (a frecuencia de $0,1 \mathrm{~Hz}$ ), muestra que existen diferencias significativas (significancia del 5,0\%) entre la viscosidad compleja de diferentes tipos de Suero (Tabla no mostrada). Sin embargo, el test de Tukey (Tabla 3) confirma que no existe diferencias significativas ente lossueros Costeños de Arjona y Turbaco, pero si se presentaron diferencias significativas entre estos dos y el Comercial y El Carmen de Bolívar, presentando la mayor viscosidad compleja este último. Igual comportamiento se presentó para la viscosidad compleja evaluada a $1,0 \mathrm{~Hz}$ y a $10,0 \mathrm{~Hz}$ (Datos no mostrados).

Tabla 3: Test de Tukey HSD de los tipos de Sueros Costeños sobre la variable respuesta viscosidad compleja a $0,1 \mathrm{~Hz}$. Parámetros del test: $\alpha=0,05$, Grados de libertad $=8$.

\begin{tabular}{|c|c|}
\hline Tipo de Suero & $\eta^{*}$ \\
\hline Turbaco & $68,06^{\mathbf{a}}$ \\
\hline Arjona & $74,05^{\mathbf{a}}$ \\
\hline Comercial & $229,86^{\mathbf{b}}$ \\
\hline El Carmen de Bolívar & $370,50^{\mathbf{c}}$ \\
\hline
\end{tabular}

\section{CONCLUSIONES}

Los Sueros que se producen con base en leche tienen mayor viscoelasticidad que los producidos a partir de lacto Suero. La pasteurización de leche acentúa el carácter viscoelástico del Suero Costeño. La viscosidad compleja resulto igual a la aparente, en todo el intervalo de velocidad de deformación y frecuencia. Los modelos que mejor describen el comportamiento viscoelástico de los Sueros Costeños son los de Ellis y Cross, mientras que el modelo de potencia, presento un buen ajuste a los datos experimentales. Todos los Sueros costeños tienen un comportamiento pseudoplástico. Los Sueros Costeños de Turbaco yArjona, tienen similar comportamiento viscoelástico,y presentan diferencia significativa con los Sueros de El Carmen de Bolívar y Comercial, los cuales también presentan diferencia significativa entre sí.

\section{REFERENCIAS}

Acevedo, D., A. Rodríguez y A. Fernández, Efecto de las variables de proceso sobre la cinética de acidificación, la viabilidad y la sinéresis del suero costeño colombiano. InformaciónTecnológica: 21(2), 29-36 (2010).

Barnes, H., A handbook of elementary rheology, $1^{\text {a }}$ Edition, 84-89, University of Wales, Institute of nonnewtonian, fluid mechanics, Cardiff, Wales, UK (2000).

Campo, I y C. Tovar, Influence of the starch content in the viscoelastic properties of surimi gels, Journal of Food Engineering: 84(1), 140-147 (2008).

Chhabra, R.P. y J.F. Richardson, Non-Newtonian Flow and Applied Rheology: Engineering Applications, $2^{\underline{a}}$ Edition, 97-101, Butterworth-Heinemann, Oxford, United States (2008).

Da Silva, J., M. Gonçalves, y A. Rao, Viscoelastic behaviour of mixtures of locust beangum and pectin dispersions, Journal of Food Engineering, 18(3), 211-228 (1993).

Freeman, J., Rheological Methods in Food Process Engineering, 2a Edition, 325-331, Freeman Press, Michigan, United States (1992).

Granados, C., D. Acevedo y R. Torres, Calidad de la leche y del suero costeño de los municipios Turbaco, Arjona y Carmen de Bolívar-Colombia, Revista Lasallista de Investigación: 9(2), 132-137 (2012).

Granados, C., G. Gonzalo y D. Acevedo, Tecnificación, caracterización fisicoquímica y microbiológica del queso de capa de Mompox (Colombia). Rev. Bio. Agro: 8(2), 440-450 (2009).

Ibarz, A. y M. Ortiz, Reología de zumos de melocotón. Influencia del contenido en pulpa, Alimentación, Equipos y Tecnología, 12(8), 81-86 (1993). 
Jena, R. y S. Bhattacharya, Viscoelastic characterization of rice gel, Journal of Texture Studies: 34(4), 349360 (2003).

Miranda, J., A.F. Guerrero y P. Pertal, Reología de derivados de la yema de huevo deshidratada, Grasas y Aceites: 51(4), 244-250 (2000).

Lucey, J. y otros tres autores, Microstructure, permeability and appearance of acid gels made from skim milk. FoodHydrocolloids, 12(2), 159-165 (1998).

Ramírez, M.A. Desarrollo de un aderezo elaborado con base en aceite de aguacate y estudio de sus propiedades fisicoquímicas y reológicas, Tesis de pregrado, Departamento de Ingeniería Química y Alimentos, Universidad de las Américas Puebla, Cholula, Puebla, México (2008).

Ramos, A. y A. Ibarz, Comportamiento viscoelástico de pulpa de membrillo en función de la concentración de sólidos solubles. Ciencia y Tecnología de Alimentos: 26(1), 214-219 (2006).

Schramm G. A Practical Approach to Rheology and Rheometry. 2nd Ed., Gebrueder HAAKE GmbH, Karlsruhe (RFA)(2000)

Sahin, S. y S. Gülüm, Physicalproperties of foods, 1르 Edition, 75-76, Springer-Verlag, New York, UnitedEstates (2006).

Shukla, A. y S.S. Rizvi, Viscoelastic properties of butter, Journal of Food Science: 60(5), 902-905 (1995).

Tabilo-Munizaga, G. y G. Barbosa-Cánovas, Rheology for the food industry, Journal of Food Engineering: $67(1-2), 147-156$ (2005). 